\title{
Maternal anxiety during pregnancy and newborn epigenome-wide
}

\section{DNA methylation}

Sara Sammallahti, + M.D., Ph.D., Andrea P Cortes Hidalgo, $\$$ M.D., M.Sc., Samuli Tuominen, M.Sc., Anni Malmberg, B.Sc., Rosa H Mulder, M.Sc., Kelly J Brunst, Ph.D., Silvia Alemany, Ph.D., Nancy S McBride, M.Sc., Paul Yousefi, Ph.D., Jonathan A Heiss, Ph.D., Nia McRae, M.P.H., Christian M Page, Ph.D., Jianping Jin, Ph.D., Giancarlo Pesce, Ph.D., Doretta Caramaschi, Ph.D., Sheryl L Rifas-Shiman, M.P.H., Nastassja Koen, Ph.D., Charleen D Adams, M.P.H., Ph.D., Maria C Magnus, Ph.D., Nour Baïz, Ph.D., Andrew Ratanatharathorn, M.A., Darina Czamara, Ph.D., Siri E Håberg, Ph.D., Elena Colicino, Ph.D., Andrea A Baccarelli, M.D., Ph.D., Andres Cardenas, Ph.D., Dawn L DeMeo, M.D., Deborah A Lawlor, M.B.Ch.B., Ph.D., Caroline L Relton, Ph.D., Janine F Felix, M.D., Ph.D., Marinus H van IJzendoorn, Ph.D., Marian J Bakermans-Kranenburg, Ph.D., Eero Kajantie, D.Med.Sc., Katri Räikkönen, Ph.D., Jordi Sunyer, Ph.D., Gemma C Sharp, Ph.D., Lotte C Houtepen, Ph.D., Ellen A Nohr, Ph.D., Thorkild I A Sørensen, D.Med.Sc., Martha M Téllez-Rojo, Ph.D., Robert O Wright, Ph.D., Isabella Annesi-Maesano, M.D., Ph.D., John Wright, M.D., Marie-France Hivert, M.D., Rosalind J Wright, M.D., M.P.H., Heather J Zar, Ph.D., Dan J Stein, M.D., Ph.D., Stephanie J London, M.D., Dr.P.H., Charlotte A M Cecil, $\$$ Ph.D., Henning Tiemeier, $\$$ M.D., Ph.D., Jari Lahti, $\$$ Ph.D. 
From the Beckman Research Institute of City of Hope, Department of Population Sciences, Duarte, CA, USA (C.D.A.), Bradford Institute for Health Research, Bradford Teaching Hospitals, NHS Foundation Trust, Bradford, UK (J.W.), Brigham and Women's Hospital, Channing Division of Network Medicine, Boston, MA, USA (D.L.D.), Centre for Fertility and Health, Norwegian Institute of Public Health, Oslo, Norway (C.M.P., S.E.H, M.C.M.), Children's Hospital, Helsinki University Hospital and University of Helsinki, Helsinki, Finland (E.K.), CIBER Epidemiología y Salud Pública (CIBERESP), Spain (S.A., J.S.), Columbia University Mailman School of Public Health, Precision Environmental Health Lab, New York, NY, USA (A.A.B.), Columbia University, Department of Epidemiology, New York City, NY, USA (A.R.), Epidemiology Branch, National Institute of Environmental Health Sciences, National Institutes of Health, Department of Health and Human Services, Research Triangle Park NC, USA (S.J.L.), Erasmus MC, University Medical Center Rotterdam, Department of Adolescent and Child Psychiatry and Psychology, Rotterdam, the Netherlands (A.P.C.H., C.A.M.C., R.H.M., S.S., H.T.), Erasmus MC, University Medical Center Rotterdam, Department of Epidemiology, Rotterdam, the Netherlands (C.A.M.C.), Erasmus MC, University Medical Center Rotterdam, Department of Pediatrics, Rotterdam, the Netherlands (J.F.F.), Erasmus MC, University Medical Center Rotterdam, Generation R Study Group, Rotterdam, the Netherlands (A.P.C.H., C.A.M.C., J.F.F., R.H.M., S.S., H.T.), Erasmus University Rotterdam, Department of Psychology, Education, and Child Studies, Rotterdam, The Netherlands (M.H.v.IJ.), Finnish Institute for Health and Welfare, Helsinki and Oulu, Finland (E.K.), Harvard Medical School, Boston, MA, USA (D.D.), Harvard Medical School, Department of Population Medicine, Harvard Pilgrim Health Care Institute, Boston, MA, USA (M.F.H., S.L.R.-S.), Harvard T.H. Chan School of Public Health, Department of 
Epidemiology, Boston, MA, USA (A.R.), Harvard T.H. Chan School of Public Health, Department of Social and Behavioral Science, Boston, MA, USA (S.S., H.T.), Icahn School of Medicine at Mount Sinai, Department of Environmental Medicine and Public Health, New York, NY, USA (E.C., J.A.H.), Icahn School of Medicine at Mount Sinai, Environmental Medicine \& Public Health, Institute for Exposomic Research, New York, NY, USA (R.J.W.), Icahn School of Medicine at Mount Sinai, New York, NY, USA (N.M., R.O.W.), INSERM UMR-S 1136, EPAR, Saint-Antoine Medical School, Paris, France (I.A.-M., N.B., G.P.), IMIM (Hospital del Mar Medical Research Institute), Barcelona, Spain (J.S.), ISGlobal, Barcelona Institute for Global Health, Barcelona, Spain (S.A., J.S.), Leiden University, Institute of Education and Child Studies, Leiden, the Netherlands (R.H.M.), Leiden University, Leiden Institute for Brain and Cognition, Leiden, The Netherlands (M.J.B.-K.), Leiden University Medical Center, Molecular Epidemiology, Department of Biomedical Data Sciences, Leiden, The Netherlands (C.C.), Max-Planck-Institute of Psychiatry, Department of Translational Research in Psychiatry, Munich, Germany (D.Cz.), Massachusetts General Hospital, Diabetes Unit, Boston, MA, USA (M.-F.H.), National Institute of Public Health, Center for Nutrition and Health Research, Cuernavaca, Mor. Mexico (M.M.T.-R.), Norwegian University of Science and Technology, Department of Clinical and Molecular Medicine, Trondheim, Norway (E.K.), Oslo Centre for Biostatistics and Epidemiology, Oslo University Hospital, Oslo, Norway (C.M.P.), Oulu University Hospital and University of Oulu, PEDEGO Research Unit, MRC Oulu, Oulu, Finland (E.K.), Sorbonne Université, Epidemiology of Allergic and Respiratory Diseases Department (EPAR), Pierre Louis Institute of Epidemiology and Public Health (IPLESP), Paris, France (N.B., G.P.), South African Medical Research Council (SAMRC) Unit on Child and Adolescent Health, Cape Town, South Africa (H.J.Z), 
South African Medical Research Council (SAMRC) Unit on Risk and Resilience in Mental Disorders, Cape Town, South Africa (N.K., D.J.S.), Universitat Pompeu Fabra (UPF), Barcelona, Spain (J.S., S.A.), University of Bristol, MRC Integrative Epidemiology Unit, Population Health Sciences, Bristol Medical School, Bristol, UK (D.Ca., L.C.H., D.A.L., M.C.M., N.S.M., C.L.R., G.C.S., T.I.A.S., P.Y.), University of California, Division of Environmental Health Sciences, School of Public Health, Berkeley, CA, USA (A.C.), University of Cambridge, School of Clinical Medicine, Cambridge, United Kingdom (M.H.v.IJ.), University of Cape Town, Department of Paediatrics and Child Health, South Africa (H.J.Z.), University of Cape Town, Department of Psychiatry and Mental Health, Cape Town, South Africa (N.K., D.J.S.), University of Cape Town, Neuroscience Institute, Cape Town, South Africa (N.K., D.J.S.), University of Cincinnati, College of Medicine, Department of Environmental Health, Cincinnati, OH, USA (K.J.B.), University of Copenhagen, Faculty of Health and Medical Sciences, Department of Public Health, Copenhagen, Denmark (T.I.A.S.), University of Copenhagen, Faculty of Medical and Health Sciences, Novo Nordisk Foundation Center for Basic Metabolic Research, Copenhagen, Denmark (T.I.A.S.), University of Helsinki, Department of Psychology and Logopedics, Helsinki, Finland (J.L., A.M., K.R., S.S., S.T.), University of Southern Denmark, Institute of Clinical Research and Department of Gynaecology and Obstetrics, Odense, Denmark (E.A.N.), Vrije Universiteit Amsterdam, Clinical Child \& Family Studies, Amsterdam, The Netherlands (M.J.B.-K.), Westat, Durham, NC, USA (J.J.)

Address correspondence to Prof Henning Tiemeier, Department of Social and Behavioral Science, Harvard T.H. Chan School of Public Health, 677 Huntington Avenue, Boston, MA 02115, USA (tiemeier@hsph.harvard.edu)

Running title: Perinatal anxiety and newborn DNA methylation 


\section{ABSTRACT}

Maternal anxiety during pregnancy is associated with adverse fetal, neonatal, and child outcomes, but biological mechanisms remain unclear. Altered fetal DNA methylation (DNAm) has been proposed as a potential underlying mechanism. In the current study, we performed a meta-analysis to examine the associations between maternal anxiety, measured prospectively during pregnancy, and genome-wide DNAm from umbilical cord blood. Sixteen non-overlapping cohorts from 12 independent longitudinal studies of the Pregnancy And Childhood Epigenetics Consortium participated, resulting in a combined dataset of 7243 mother-child dyads. We examined prenatal anxiety in relation to genome-wide DNAm and differentially methylated regions. We observed no association between the general symptoms of anxiety during pregnancy or pregnancyrelated anxiety, and DNAm at any of the CpG sites, after multiple-testing correction. Further, we identify no differentially methylated regions associated with maternal anxiety. At the cohort-level, of the 21 associations observed in individual cohorts, none replicated consistently in the other cohorts. In conclusion, contrary to some previous studies proposing cord blood DNAm as a promising potential mechanism explaining the link between maternal anxiety during pregnancy and adverse outcomes in offspring, we found no consistent evidence for any robust associations between maternal anxiety and DNAm in cord blood. Larger studies and analysis of DNAm in other tissues may be needed to establish subtle or subgroup-specific associations between maternal anxiety and the fetal epigenome. 


\section{INTRODUCTION}

Anxiety symptoms during pregnancy are common, and an estimated $15 \%$ of pregnant women fulfill the diagnostic criteria of any anxiety disorder. ${ }^{1}$ Perinatal anxiety may not only affect the mother, but could also have long-lasting implications for the offspring. Children of mothers with high levels of prenatal anxiety are more likely to be preterm and small-for-gestational-age, and to have emotional and behavioral problems that may persist beyond childhood. ${ }^{2-4}$

The biological pathways underlying maternal mental health during pregnancy and offspring development are poorly understood. One proposed pathway is fetal programming through epigenetic modifications: prenatal stressors may alter fetal DNA methylation (DNAm), which may affect downstream gene expression, neuroendocrine functioning, and behavioral development. ${ }^{5}$ Several animal studies have supported this hypothesis. ${ }^{5}$ However, human evidence of associations between maternal anxiety during pregnancy and offspring DNAm is mixed and mostly based on small samples. ${ }^{6}$ A recent epigenome-wide association study (EWAS) found that high levels of prenatal anxiety predicted differential DNAm at $13 \mathrm{CpG}$ sites in cord blood $(\mathrm{N}=445)$, but these findings did not replicate in another sample $(\mathrm{N}=969) .{ }^{14}$

We present a meta-analysis of epigenome-wide associations between prenatal generaland pregnancy-related anxiety and cord blood DNAm, from 12 independent longitudinal studies (total $\mathrm{N}=7,243$ ). 


\section{MATERIALS AND METHODS}

\section{Participating studies}

Sixteen cohorts from 12 independent longitudinal studies, in the Pregnancy And Childhood Epigenetics Consortium (PACE), ${ }^{15}$ participated in this meta-analysis. These were the 1) Avon Longitudinal Study of Parents and Children (ALSPAC) (United Kingdom), ${ }^{16}$ 2) Born in Bradford (BiB) (United Kingdom), ${ }^{17}$ subdivided into South Asian (BiB-1) and European (BiB-2) cohorts, 3) Drakenstein Child Health Study (DCHS) (South Africa), ${ }^{18}$ comprising the DCHS-450k and DCHS-EPIC cohorts, differentiated by the choice of DNAm array (Illumina 450k and EPIC, respectively), 4) EDEN Mother-Child Cohort (EDEN) (France), ${ }^{19}$ 5) Generation R Study (Generation R) (Netherlands), ${ }^{20}$ 6) Genome-Wide Population-based Association Study of Extremely Overweight Young Adults (GOYA) (Denmark; weight distribution of the subsample used for these analyses corresponds to the general population), ${ }^{21}$ 7) INfancia y Medio Ambiente (INMA) (Spain), ${ }^{22}$ 8) Norwegian Mother, Father and Child Cohort Study (MoBa) (Norway), ${ }^{23}$ comprising the MoBa-1 and the MoBa-2 cohorts, two independent samples from the MoBa study, 9) Prediction and Prevention of Preeclampsia and Intrauterine Growth Restriction study (PREDO) (Finland), ${ }^{24}$ comprising the PREDO450k and the PREDO-EPIC cohort, differentiated by Illumina 450k vs EPIC DNAm array, respectively, 10) Programming Research in Obesity, Growth, Environment and Social Stressors study (PROGRESS) (Mexico), ${ }^{25}$ 11) PRogramming of Intergenerational Stress Mechanisms study (PRISM) (USA), ${ }^{26}$ and 12) Project Viva (Viva) (USA). ${ }^{27}$

See Supplementary methods and PACE profile ${ }^{15}$ for details. 


\section{Measures}

Exposure: Maternal anxiety during pregnancy

Anxiety can be assessed using general self-report instruments or instruments specifically designed for pregnant women, which may be more predictive of perinatal outcomes and include pregnancy-related items such as childbirth-related worries. ${ }^{28} \mathrm{We}$ examined the two separately: we refer to anxiety symptoms measured using general instruments as general anxiety (not to be confused with generalized anxiety disorder), and pregnancy-related anxiety symptoms measured using pregnancy-specific instruments as pregnancy-related anxiety. In one cohort (INMA), mothers reported, during prenatal interviews, if a physician had diagnosed them with any anxiety disorder: we included INMA in the primary meta-analysis of general anxiety (Table 1). Instruments are described in Supplementary methods.

Each cohort dichotomized questionnaire scores, contrasting the highest-scoring $15 \%$ of mothers ("high-anxiety" group), against other mothers. ${ }^{1,4}$ Scores were neither transformed nor standardized. In sensitivity analyses, we used continuous questionnaire scores (winsorized at +/- 3SD): higher scores reflect more symptoms. Cohorts with repeated measures of anxiety during pregnancy calculated mean scores (Table 1).

\section{Outcome: DNAm measurements}

Newborn umbilical cord blood DNAm was assessed with the Illumina ${ }^{\circledR}$ HumanMethylation450 (450k) or HumanMethylationEPIC (EPIC) BeadChip assay at Illumina or cohort-specific laboratories. Cohorts performed sample processing, quality control and normalization as described in Supplementary methods. 
We used normalized, untransformed beta values, ranging from 0 (completely unmethylated) to 1 (completely methylated), after trimming extreme outliers (3xinterquartile range from the quartile limit).

We excluded probes mapped to $\mathrm{X} / \mathrm{Y}$ chromosomes, polymorphic CpGs (overlapping with known single-nucleotide-polymorphisms [SNPs]), ${ }^{29}$ control or cross-reactive probes (targeting repetitive sequences/co-hybridizing to alternate sequences). ${ }^{30,31}$

Function-related information was derived from GeneCards and GWAS Catalog (https://www.genecards.org; https://www.ebi.ac.uk/gwas, accessed August 17 $7^{\text {th }}, 2020$ ).

\section{Covariates}

Based on theoretical expectations, ${ }^{3}$ we adjusted for child sex, maternal age, socioeconomic status (SES, maternal education/family income), cell counts, ethnicity/ancestry (if applicable) and technical covariates (e.g. batch) (Supplementary methods). We used reference-based projection/quadratic programming to estimate cell type proportions: ${ }^{32,33}$ EDEN used the Houseman reference panel, ${ }^{32}$ PRISM a combined panel, ${ }^{33,34}$ and others the Bakulski panel. ${ }^{33}$

In a sensitivity analysis, we added the maternal smoking covariate. This was a separate model because causal associations between smoking and anxiety could be bidirectional and smoking could mediate some effects on DNAm (Supplementary methods).

\section{Statistical analyses}

Cohort-specific EWAS

Cohort-level EWASes were performed according to a predefined analysis plan. We used robust multiple linear regression ( $r l m ; M A S S$ R-package) to control for potential 
heteroscedasticity and influential DNAm outliers. We included participants with complete information. Cohorts excluded known chromosomal abnormalities, multiple births, and one random sibling per sibling pair. Models are described in Supplementary methods.

Primary meta-analysis

We examined associations between maternal general anxiety during pregnancy and DNAm at 364,659 CpG sites across 15 cohorts (ALSPAC, BiB-1, BiB-2, DCHS-450k, DCHS-EPIC, EDEN, Generation R, GOYA, INMA, MoBa-1, MoBa-2, PREDO-450k, PREDO-EPIC, PRISM, PROGRESS). We combined 450k and EPIC data and only included sites that are available on the $450 \mathrm{k}$. We performed a sample-size-weighted meta-analysis using $R$ 3.6.1 (https://www.r-project.org/) and METAL (version released 2018-08-28), ${ }^{35}$ choosing the sample-size-weighted approach because of variation across anxiety scales in different cohorts. Probes were annotated using meffil (hg19/b37). ${ }^{29}$

To assess epigenome-wide statistical inflation, we calculated cohort- and meta-analysislevel genomic inflation factor lambdas $(\lambda)$ and examined quantile-quantile plots.

Cohort-level results were meta-analyzed at Erasmus MC; a shadow meta-analysis was conducted independently at the University of Helsinki.

Secondary meta-analyses

We performed two secondary meta-analyses. First, we examined associations between pregnancy-related anxiety and DNAm (Generation R, PRISM, PROGRESS, Viva); second, associations between general anxiety and DNAm at the 439,294 EPIC-assayspecific CpGs (BiB-1, BiB-2, DCHS-EPIC, PROGRESS). 


\section{Multiple-testing correction}

All reported p-values are two-sided. For multiple-testing correction, we used Bonferroni-correction based on the number of probes (primary meta-analysis cut-off $\left.\mathrm{p}<1.37 \times 10^{-7}\right)$. Additionally, we examined our results using the Benjamini Hochberg False-Discovery-Rate method (FDR). ${ }^{36}$ When we observed no statistically significant associations, we used a suggestive $\mathrm{p}$-value threshold $\left(\mathrm{p}<5 \times 10^{-5}\right)$ to select $\mathrm{CpGs}$ for follow-up analyses.

\section{Sensitivity analyses}

We repeated the primary and secondary meta-analyses twice: first, adding smoking into covariates, and second, modelling anxiety as continuous. To address potential acrossstudy heterogeneity, we examined $\mathrm{I}^{2}$ statistics, and repeated the primary meta-analysis only including cohorts with 1) 450k or 2) EPIC array, 3) predominantly Europeanancestry cohorts (ALSPAC, BiB-2, EDEN, Generation R, GOYA, INMA, MoBa, PREDO), and 4) cohorts using the Bakulski panel for cell type correction. ${ }^{33}$

We identified the 30 probes with the largest effect estimates for general anxiety in the three largest cohorts (Generation R, MoBa-1, ALSPAC; 10 probes/cohort), and examined whether effects were consistent across cohorts. This was repeated with pregnancy-related anxiety (largest cohorts: Generation R, Viva, PROGRESS).

\section{DMR analysis}

To identify differentially methylated regions (DMRs), we used $d m r f f:{ }^{37}$ it addresses the correlation structure of DNAm and can be used to identify DMRs at the meta-analytic level. Ten cohorts (ALSPAC, EDEN, GOYA, Generation R, INMA, MoBa-1, PREDO450k, PREDO-EPIC, PRISM, PROGRESS) calculated correlations between each CpG 
and the next $20 \mathrm{CpGs}$ downstream. We used data on $\mathrm{CpGs}$ covered by $450 \mathrm{k}$. We identified candidate regions: sets of nearby $\mathrm{CpG}$ sites $(\leq 500 \mathrm{bp}$ between consecutive sites) that associated with anxiety (nominal $\mathrm{p}$-values $<0.05$ in cohort-level EWAS, effect estimates in the same direction), and, using an extension of inverse-variance weighted meta-analysis, calculated the $d m r f f$ statistics. We then meta-analyzed those statistics by selecting sub-regions with the most extreme meta-analysis statistic. P-values for candidate regions were Bonferroni-corrected.

\section{Blood-brain comparisons}

To examine correlation between blood and brain DNAm levels at CpG sites with metaanalytical $\mathrm{p}<5 \times 10-5$, we used two tools. The first by Hannon et $\mathrm{al}^{38}$ reports correlations between DNAm in blood vs prefrontal cortex, entorhinal cortex, superior temporal gyrus, and cerebellum. Secondly, BECon shows correlations between DNAm in blood vs parietal, anterior prefrontal, and ventral temporal cortex (Brodmann 7, 10, 20, respectively). ${ }^{39}$

\section{Code and data availability and ethical statements}

All studies acquired approval from local ethics committees and informed consent from participants. Site-level meta-analytical results are available at [the EWAS Catalog, upon publication]. For access to cohort-level data, requests can be sent directly to individual studies. Analytical codes can be requested from authors. 


\section{RESULTS}

\section{Study characteristics}

Table 2 describes our 7,243 mother-child dyads. Supplementary Table 1 shows characteristics stratified by low-vs-high anxiety.

\section{General anxiety during pregnancy and DNAm}

We observed no associations between maternal general anxiety during pregnancy and DNAm of 364,659 CpG sites in cord blood, among 6,686 mother-child dyads, when using Bonferroni correction $\left(\mathrm{p}<1.37 \times 10^{-7}\right)$ (Figure 1A). We found no evidence of genomic inflation ( $\lambda=0.96$, quantile-quantile plot in Figure 1B).

In Table 3, we list the $15 \mathrm{CpG}$ sites that were most strongly associated $\left(\mathrm{p}<5 \times 10^{-5}\right)$ with maternal general anxiety during pregnancy. Figure 2 shows a regression coefficient forest-plot at these $15 \mathrm{CpG}$ sites across cohorts: effect direction was not consistent across cohorts for any of these sites.

\section{Secondary meta-analyses}

Pregnancy-related anxiety

We found no association between pregnancy-related anxiety and DNAm among the 2,089 mother-child dyads in four cohorts with available data (Figure SF1, $\lambda=0.92$ ). Figure SF2 shows the $8 \mathrm{CpG}$ sites that were most strongly associated $\left(\mathrm{p}<5 \times 10^{-5}\right)$ with pregnancy-related anxiety and the variation in estimates across cohorts. At two of these sites (cg18769357, $\operatorname{cg} 03985478)$, all four cohorts reported the association in the same direction: for $c g 18769357$, these associations were only nominally significant $(\mathrm{p}<0.05)$ in Generation R and PRISM; for cg03985478, they were only nominally significant in 
Generation R. Only one site, $c g 02624770$, had a nominally significant association with pregnancy-related anxiety across the three largest cohorts $(\mathrm{N}>200)$ : the direction was negative suggesting hypomethylation. None of the sites most strongly associated $\left(\mathrm{p}<5 \times 10^{-5}\right)$ with pregnancy-related anxiety overlapped with the sites most strongly associated $\left(\mathrm{p}<5 \times 10^{-5}\right)$ with general anxiety (Supplementary Table 2).

\section{EPIC-only sites}

We found no association between general anxiety during pregnancy and DNAm at the 439,294 CpG sites that are included only on the EPIC array among 1,221 mother-child dyads from four cohorts (Figure SF3, $\lambda=1.02$ ). Figure SF4 shows all 40 EPIC-only sites that were most strongly $\left(\mathrm{p}<5 \times 10^{-5}\right)$ associated with general anxiety. We identified 26 sites where general anxiety during pregnancy associated with hypermethylation across all four cohorts, and 3 sites where it associated with hypomethylation. (Figure SF4). Five sites (cg02306798, cg20639127, cg20817245, cg21202789, cg22566694) had a nominally significant $(\mathrm{p}<0.05)$ association in the same direction (all positive) with general anxiety across the three largest cohorts (N>200) (Figure SF4).

\section{FDR}

We additionally applied the FDR instead of Bonferroni method to correct for multiple testing. We identified no associations passing this more lenient threshold in the primary, nor the secondary meta-analyses.

\section{Sensitivity analyses}

We examined results after cohorts additionally adjusted for maternal smoking during pregnancy (all cohorts), or when treating the exposure as continuous (available in 14 cohorts, maximum $\mathrm{N}=6,350$ ) and identified no associations between general or 
pregnancy-related anxiety and DNAm at any of the 450k or EPIC-assay sites (corrected for multiple testing).

We repeated the primary meta-analysis in cohorts with 1) 450k (maximum $\mathrm{N}=5,345$ ), 2) EPIC (maximum $\mathrm{N}=1,341$ ), 3) predominantly European participants (maximum $\mathrm{N}=5,581)$, and 4) Bakulski panel ${ }^{33}(\mathrm{~N}=6,380)$. Again, no associations were observed.

Figure SF5 illustrates associations between DNAm and general (panel A) and pregnancy-related (B) anxiety, for 30 probes with the largest effect estimates in the largest cohorts. We found no consistent associations across cohorts at these sites.

\section{DMR analysis}

We identified no DMRs associated with general/pregnancy-related anxiety (after Bonferroni correction) (Supplementary Table 3).

\section{Blood-brain comparisons}

Among CpGs closest to statistical significance, none showed consistent, wide-spread correlations between blood and brain DNAm.

Figure SF6 shows that two of the 15 sites most strongly associated $(p<5 \times 10-5)$ with general anxiety (cg13516541, cg01987516) exhibited a relatively concordant DNAm pattern $(\mathrm{r}>0.41, \mathrm{p}<0.001)$ across blood vs the prefrontal cortex and superior temporal gyrus. ${ }^{38}$ Two of the 8 sites most strongly associated with pregnancy-related anxiety (cg01093369, cg03985478) showed high correlation between blood vs brain DNAm $(\mathrm{r}>0.44, \mathrm{p}<0.001){ }^{38}$

Figure SF7 shows that among the sites most strongly related to general anxiety, one (cg14776033) showed moderate negative correlations between blood and the brain 
regions using BECon $(\mathrm{r}=-0.51 \ldots-0.31) .{ }^{39}$ One site among those most strongly associated with pregnancy-related anxiety (cg14200609) showed moderate negative correlations between blood and all brain regions $(\mathrm{r}=-0.53 \ldots-0.26)$.

\section{Sites of interest: look-up}

Supplementary Table 4 shows meta-analytical results for CpGs associated with maternal anxiety/depression in a previous EWAS, ${ }^{40}$ and CpGs annotated to genes of interest for perinatal mental health epigenetics (NR3C1, NR3C2, FKBP5, HSD11B2, OXTR). e.g. 6,13,41 None were consistently associated with maternal anxiety in our metaanalysis (non-corrected p-values $>0.001$, inconsistent direction of effects).

\section{Cohort-level results}

Supplementary Table 5 shows lambdas and Bonferroni-corrected cohort-level hits. At genome-wide level, we observed 12 significant cohort-level associations between maternal general anxiety and DNAm. Five of these sites map to genes associated with neurodevelopmental/mental health-related phenotypes (cg06113534/DSCC1, cg06861562/C5orf43, cg15475427/BMP8A, cg19614454/REPS1, cg12670525/PARP6), however none of the 12 cohort-level hits overlapped across two or more cohorts, nor was the effect direction consistent across all cohorts for any of these sites.

We found 9 cohort-level associations between pregnancy-related anxiety and DNAm (Supplementary Table 5). At only one of these sites, cg19660243, effect estimates were in the same direction (positive) in all cohorts. Two sites mapped to genes previously linked to neurodevelopmental/mental health phenotypes 
(cg11920519/MAP1LC3A, cg15401317/NARS2), but showed no consistent pattern of findings across cohorts.

We advise a very cautious interpretation of these 21 cohort-level associations. They are likely false positives, given the lack of consistency across cohorts.

\section{DISCUSSION}

We found no robust evidence of associations between cord blood DNAm and either general symptoms of anxiety during pregnancy or pregnancy-related anxiety across 16 non-overlapping cohorts within 12 independent longitudinal studies. These results were consistently supported by a comprehensive set of additional analyses.

We dichotomized exposures to address different questionnaire score distributions, harmonize questionnaire-based with diagnosis-based measures, and define a subgroup of interest across a continuum of symptom severity. The $15 \%$ cut-off was validated against diagnostic interviews in our largest cohort, ${ }^{4}$ and is in line with estimated global prevalence of prenatal anxiety disorders $(\sim 15 \%),{ }^{1}$ and previous studies in non-clinical populations. ${ }^{42,43}$ Nonetheless, by modelling anxiety with a dichotomous approach, we inevitably lose information and power. Thus, we also examined anxiety symptoms as continuous scores (excluding INMA, for which only a binary diagnosis was available). Consistent with our main results, these analyses revealed no associations between anxiety and DNAm.

DNAm at nearby sites is correlated, and there is disagreement over the optimal method of genome-wide correction. We confirmed our findings using FDR and Bonferroni methods. No meta-analytical associations would survive correction for multiple testing even if we used a more lenient 450k-array P-value cut-off $(2.4 \times 10-7) .{ }^{44}$ Removal of 
invariable probes could reduce the number of tests. However, invariable probes differ per tissue and we are not aware of a cord-blood-specific reference list. Our results would not change if we filtered out across-tissue invariable probes $(n=42,315) .{ }^{45}$ We show the meta-analytic results for sites closest to the Bonferroni threshold, to facilitate evaluating across-cohort consistency. To further address non-independence between $\mathrm{CpGs}$, we searched for DMRs and found none. Finally, we uploaded the meta-analytical results for all probes in a public repository, to enable others to examine specific sites or apply future filtering methods.

We combined data from different countries and different arrays. Overall DNAm patterns are, however, highly correlated between the $450 \mathrm{k}$ and EPIC arrays. ${ }^{46}$ Sensitivity analyses did not suggest that array differences would represent a source of substantial bias. Similarly, we ran sensitivity analyses including only the predominantly European cohorts to address potential issues arising from ethnic variation. The results were unchanged.

These findings are in line with some, ${ }^{7,14}$ but not all previous studies..$^{8-10,12,13}$ One epigenome-wide study used Viva $(\mathrm{N}=445)$ as a discovery and Generation $\mathrm{R}(\mathrm{N}=969)$ as a replication sample (both studies included in this meta-analysis), ${ }^{14}$ while others relied on smaller samples from individual cohorts $(\mathrm{N}=45-576)$.

A possible interpretation of our results is that maternal symptoms of anxiety may not substantially affect circulating fetal DNAm, and the associations between prenatal anxiety and child phenotypes that have been observed in several studies ${ }^{2,3}$ are explained by other mechanistic pathways. These could include, for example, the postnatal extension of maternal anxiety and subsequent differences in parenting and the home environment, increased risk of perinatal complications/interventions, or confounding by 
genetic and environmental risk factors which could affect both anxiety and child outcomes. Anxiety may also impact methylation in specific target tissues only without reflection of these changes in blood.

Alternative interpretations may be considered in light of our study limitations. First, maternal anxiety may associate with cord blood DNAm, but the effects at individual sites could be so subtle that using the current methods, they could not be detected. With > 7,000 mother-child dyads, our study is substantially larger than previous studies, ${ }^{11,14}$ and meta-analyses of similar/smaller samples have shown robust associations between cord blood DNAm and other prenatal exposures such as maternal smoking, ${ }^{47}$ however we may have insufficient power to detect all meaningful change in DNAm. DNAm variation is site-specific, and while some evidence has suggested associations between cord blood DNAm and child neurodevelopmental outcomes, ${ }^{48}$ more information about the magnitude of associations between cord blood DNAm and relevant child phenotypes is needed before deciding what constitutes a meaningful change in DNAm. In particular, analyses of pregnancy-related anxiety were based on a smaller sample and could be underpowered. Although we observed no meta-analytic association between pregnancyrelated anxiety and cord blood DNAm, some CpGs exhibited nominal-level associations with pregnancy-related anxiety in the largest cohorts. Similarly, we report some nominal associations between general anxiety and DNAm at sites included only on the EPIC array across the largest cohorts. These could be of interest for future studies.

Second, there may be subgroup-specific associations between maternal anxiety and DNAm that merit future research. Effects specific to most severe or certain types of anxiety could go unnoticed in our population-based cohorts due to rarity/selective attrition. We lacked power to examine trait-anxiety as a separate construct ${ }^{49}$ or effects mediated by medication. Furthermore, measurement error and variation across cohorts 
in the timing and instrument used to measure anxiety likely contributed to reduced power. However, questionnaires were chosen per cohort to reliably measure anxiety within the specific population, and it is unlikely that instrument differences would hide robust, wide-spread associations between anxiety and DNAm. Most scales were previously validated in perinatal settings. ${ }^{\text {e.g. }}$ 4,28,50-52 For example, GHQ, BSI, and STAI were validated against perinatal diagnostic interviews, ${ }^{4,50}$ and CCEI correlates highly with STAI-S/STAI-T during pregnancy $\left(r=.70 / r=.76\right.$, respectively) ${ }^{52}$ (Supplementary Methods contains more psychometric information). Further, we examined pregnancyrelated anxiety separately as recommended. ${ }^{28}$ Nonetheless, some symptom- or disorderspecific effects may have gone unnoticed. Further, we encourage research into potential sex differences. We included sex as a covariate but did not examine sex-specificity, to limit the number of tests to those with the most power.

Our choice to focus on anxiety alone may be considered another limitation. For example, we did not examine comorbidity between anxiety and depression, which is common and could increase the risk for poor perinatal outcomes compared to anxiety alone. ${ }^{53} \mathrm{We}$ encourage research on related prenatal exposures, including depression, stressful events, and substance use disorder, for which the existing small epigenetic studies have shown mixed results. e.g. 14,54

Finally, emerging cell-type correction methods could prove useful. However, we used a validated approach to address cell composition, and 14 of 16 cohorts used the same reference panel. ${ }^{33}$ A study assessing prenatal phthalate exposure and cord blood DNAm found only small differences when three different cell-type reference panels were used. ${ }^{55}$ Also, no significant results emerged in analyses only including cohorts with the Bakulski panel. ${ }^{33}$ Different reference panels were, thus, unlikely to strongly influence our results. 
Our study has several strengths. Firstly, the collaboration across studies allowed us to examine the association between maternal anxiety and cord blood DNAm among $>$ 7,000 mother-child dyads. Secondly, we examined pregnancy-related anxiety, which may be more closely associated with perinatal outcomes, compared to general anxiety symptoms. ${ }^{28}$ Thirdly, combining data from several cohorts with the EPIC array, we examined DNAm across hundreds of thousands of sites that have not been examined before, in particular increasing coverage of regulatory regions. Our null findings do not definitely refute a link between prenatal anxiety and epigenetic programming. Future studies of other tissues of interest, such as the brain and the placenta, and less wellunderstood forms of epigenetic alterations, such as histone modifications, could prove relevant. Also, studies with larger samples and higher resolution could elucidate subtle effects.

Conclusions: This meta-analysis suggests little evidence of a relation between maternal anxiety during pregnancy and differential DNAm in cord blood. While this is a considerably large study on maternal mental health and offspring DNAm, even larger studies with validated clinical diagnoses instead of self-reported symptoms may be needed to establish subtle or subgroup-specific associations between maternal anxiety and the fetal epigenome. Other tissues, epigenetic mechanisms, and aspects of perinatal mental health warrant exploration, as we begin to disentangle the many biological mechanisms that could explain how perinatal maternal mental health affects the next generation.

\section{ACKNOWLEDGEMENTS}

Acknowledgements for each of the participating studies are listed in the Funding and Acknowledgements supplement. 


\section{CONFLICT OF INTEREST}

One author (Dan J Stein) received research grants and/or consultancy honoraria from Lundbeck and Sun; the other authors confirm they have no financial relationships with commercial interests to disclose. Funding for each of the participating studies is listed in the Funding and Acknowledgements supplement. There was no editorial direction or censorship from the sponsors. 


\section{REFERENCES}

1. Dennis CL, Falah-Hassani K, Shiri R. Prevalence of antenatal and postnatal anxiety: Systematic review and meta-analysis. Br J Psychiatry. 2017;210(5):315323.

2. Grigoriadis S, Graves L, Peer M, Mamisashavili L, Tomlinson G, Vigod SN, et al. Maternal anxiety during pregnancy and the association with adverse perinatal outcomes: Systematic review and meta-analysis. J Clin Psychiatry. 2018;79(5): $17 \mathrm{r} 12011$.

3. Stein A, Pearson RM, Goodman SH, Rapa E, Rahman A, McCallum M, et al. Effects of perinatal mental disorders on the fetus and child. Lancet. 2014;384:1800-1819.

4. Henrichs J, Schenk JJ, Roza SJ, van den Berg MP, Schmidt HG, Steegers EAP, et al. Maternal psychological distress and fetal growth trajectories: The Generation R study. Psychol Med. 2010;40(4):633-643.

5. Cao-Lei L, Rooij SR De, King S, Matthews SG, Metz GAS, Roseboom TJ, et al. Prenatal stress and epigenetics. Neurosci Biobehav Rev. In press.

6. Ryan J, Mansell T, Fransquet P, Saffery R. Does maternal mental well-being in pregnancy impact the early human epigenome? Epigenomics. 2017;9(3):313322.

7. Mansell T, Vuillermin P, Ponsonby AL, Collier F, Saffery R, Ryan J. Maternal mental well-being during pregnancy and glucocorticoid receptor gene promoter methylation in the neonate. Dev Psychopathol. 2016;28(4):1421-1430. 
8. Mansell T, Novakovic B, Meyer B, Rzehak P, Vuillermin P, Ponsonby A-L, et al. The effects of maternal anxiety during pregnancy on IGF2 / H19 methylation in cord blood. Transl Psychiatry. 2016;6:e765.

9. Chen J, Li Q, Rialdi A, Mystal E, Ly J, Finik J, et al. Influences of maternal stress during pregnancy on the epigenome: Comparison of placenta and umbilical cord blood. J Depress Anxiety. 2014;3(2):152.

10. Vangeel EB, Izzi B, Hompes T, Vansteelandt K, Lambrechts D, Freson K, et al. DNA methylation in imprinted genes IGF2 and GNASXL is associated with prenatal maternal stress. Genes, Brain Behav. 2015;14(8):573-582.

11. Vangeel EB, Pishva E, Hompes T, van den Hove D, Lambrechts D, Allegaert K, et al. Newborn genome-wide DNA methylation in association with pregnancy anxiety reveals a potential role for GABBR1. Clin Epigenetics. 2017;9:107.

12. Oberlander TF, Weinberg J, Papsdorf M, Grunau R, Misri S, Devlin AM. Prenatal exposure to maternal depression, neonatal methylation of human glucocorticoid receptor gene (NR3C1) and infant cortisol stress responses. Epigenetics. 2008;3(2):97-106.

13. Hompes T, Izzi B, Gellens E, Morreels M, Fieuws S, Pexters A, et al. Investigating the influence of maternal cortisol and emotional state during pregnancy on the DNA methylation status of the glucocorticoid receptor gene (NR3C1) promoter region in cord blood. J Psychiatr Res. 2013;47(7):880-891.

14. Cardenas A, Faleschini S, Cortes Hidalgo A, Rifas-Shiman SL, Baccarelli AA, DeMeo DL, et al. Prenatal maternal antidepressants, anxiety, and depression and offspring DNA methylation: Epigenome-wide associations at birth and 
persistence into early childhood. Clin Epigenetics. 2019;11(1):56.

15. Felix JF, Joubert BR, Baccarelli AA, Sharp GC, Almqvist C, Annesi-Maesano I, et al. Cohort profile: Pregnancy and childhood epigenetics (PACE) consortium. Int J Epidemiol. 2018;47(1):22-23.

16. Fraser A, Macdonald-Wallis C, Tilling K, Boyd A, Golding J, Davey Smith G, et al. Cohort profile: The Avon longitudinal study of parents and children: ALSPAC mothers cohort. Int J Epidemiol. 2013;42(1):97-110.

17. Wright J, Small N, Raynor P, Tuffnell D, Bhopal R, Cameron N, et al. Cohort profile: The Born in Bradford multi-ethnic family cohort study. Int J Epidemiol. 2013;42(4):978-991.

18. Stein DJ, Koen N, Donald KA, Adnams CM, Koopowitz S, Lund C, et al. Investigating the psychosocial determinants of child health in Africa: The Drakenstein Child Health Study. J Neurosci Methods. 2015;252:27-35.

19. Heude B, Forhan A, Slama R, Douhaud L, Bedel S, Saurel-Cubizolles M-J, et al. Cohort Profile: The EDEN mother-child cohort on the prenatal and early postnatal determinants of child health and development. Int $J$ Epidemiol. 2016;45(2):353-363.

20. Kooijman MN, Kruithof CJ, van Duijn CM, Duijts L, Franco OH, van IJzendoorn MH, et al. The Generation R Study: Design and cohort update 2017. Eur J Epidemiol. 2016;31(12):1243-1264.

21. Paternoster L, Evans DM, Aagaard Nohr E, Holst C, Gaborieau V, Brennan P, et al. Genome-wide population-based association study of extremely overweight young adults - the GOYA study. PLoS One. 2011;6(9):e24303. 
22. Guxens M, Ballester F, Espada M, Fernández MF, Grimalt JO, Ibarluzea J, et al. Cohort profile: The INMA-INfancia y Medio Ambiente-(environment and childhood) project. Int J Epidemiol. 2012;41(4):930-940.

23. Magnus P, Birke C, Vejrup K, Haugan A, Alsaker E, Daltveit AK, et al. Cohort profile update: The Norwegian Mother and Child Cohort Study (MoBa). Int J Epidemiol. 2016;45(2):382-388.

24. Girchenko P, Lahti M, Tuovinen S, Savolainen K, Lahti J, Binder EB, et al. Prediction and Prevention of Preeclampsia and Intrauterine Growth Restriction (PREDO) study. Int J Epidemiol. 2017;46(5):1380-1381.

25. Tamayo y Ortiz M, Téllez-Rojo MM, Trejo-Valdivia B, Schnaas L, OsorioValencia E, Coull B, et al. Maternal stress modifies the effect of exposure to lead during pregnancy and 24-month old children's neurodevelopment. Environ Int. 2017;98:191-197.

26. Brunst KJ, Enlow MB, Kannan S, Carroll KN, Coull BA, Wright RJ. Effects of prenatal social stress and maternal dietary fatty acid ratio on infant temperament: Does race matter? Epidemiol. 2014;4(4):1000167.

27. Oken E, Baccarelli AA, Gold DR, Kleinman KP, Litonjua AA, De Meo D, et al. Cohort profile: Project Viva. Int J Epidemiol. 2015;44:37-48.

28. Brunton RJ, Dryer R, Saliba A, Kohlhoff J. Pregnancy anxiety: A systematic review of current scales. J Affect Disord. 2015;176:24-34.

29. Min JL, Hemani G, Davey Smith G, Relton C, Suderman M. Meffil: Efficient normalization and analysis of very large DNA methylation datasets. Bioinformatics. 2018;34(23):3983-3989. 
30. Chen YA, Lemire M, Choufani S, Butcher DT, Grafodatskaya D, Zanke BW, et al. Discovery of cross-reactive probes and polymorphic CpGs in the Illumina Infinium HumanMethylation450 microarray. Epigenetics. 2013;8(2):203-209.

31. McCartney DL, Walker RM, Morris SW, McIntosh AM, Porteous DJ, Evans KL. Identification of polymorphic and off-target probe binding sites on the Illumina Infinium MethylationEPIC BeadChip. Genomics Data. 2016;9:22-24.

32. Houseman EA, Accomando WP, Koestler DC, Christensen BC, Marsit CJ, Nelson $\mathrm{HH}$, et al. DNA methylation arrays as surrogate measures of cell mixture distribution. BMC Bioinformatics. 2012;13:86.

33. Bakulski KM, Feinberg JI, Andrews SV, Yang J, Brown S, McKenney SL, et al. DNA methylation of cord blood cell types: Applications for mixed cell birth studies. Epigenetics. 2016;11(5):354-362.

34. de Goede OM, Razzaghian HR, Price EM, Jones MJ, Kobor MS, Robinson WP, et al. Nucleated red blood cells impact DNA methylation and expression analyses of cord blood hematopoietic cells. Clin Epigenetics. 2015;7:95.

35. Willer CJ, Li Y, Abecasis GR. METAL: Fast and efficient meta-analysis of genomewide association scans. Bioinformatics. 2010;26:2190-2191.

36. Benjamini Y, Hochberg Y. Controlling the False Discovery Rate: A practical and powerful approach to multiple testing. J R Stat Soc Ser B. 1995;57(1):289-300.

37. Suderman M, Staley JR, French R, Arathimos R, Simpkin A, Tilling K. Dmrff: Identifying Differentially Methylated Regions efficiently with power and control. BioRxiv. Preprint. doi:10.1101/508556 
38. Hannon E, Lunnon K, Schalkwyk L, Mill J. Interindividual methylomic variation across blood, cortex, and cerebellum: Implications for epigenetic studies of neurological and neuropsychiatric phenotypes. Epigenetics. 2015;10(11):10241032.

39. Edgar RD, Jones MJ, Meaney MJ, Turecki G, Kobor MS. BECon: A tool for interpreting DNA methylation findings from blood in the context of brain. Transl Psychiatry. 2017;7:e1187.

40. Non AL, Binder AM, Kubzansky LD, Michels KB. Genome-wide DNA methylation in neonates exposed to maternal depression, anxiety, or SSRI medication during pregnancy. Epigenetics. 2014;9(7):964-972.

41. Conradt E, Lester BM, Appleton AA, Amstrong DA, Marsit CJ. The roles of DNA methylation of $\mathrm{NR} 3 \mathrm{C} 1$ and $11 \beta-\mathrm{HSD} 2$ and exposure to maternal mood disorder in utero on newborn neurobehavior. 2013;8(12):1321-1329.

42. Heron J, O’Connor TG, Evans J, Golding J, Glover V, ALSPAC Study Team. The course of anxiety and depression through pregnancy and the postpartum in a community sample. J Affect Disord. 2004;80(1):65-73.

43. Glover V, O’Connor TG, Heron J, Golding J, ALSPAC Study Team. Antenatal maternal anxiety is linked with atypical handedness in the child. Early Hum Dev. 2004;79(2):107-118.

44. Saffari A, Silver MJ, Zavattari P, Moi L, Columbano A, Meaburn EL, et al. Estimation of a significance threshold for epigenome-wide association studies. Genet Epidemiol. 2018;42(1):20-33.

45. Edgar RD, Jones MJ, Robinson WP, Kobor MS. An empirically driven data 
reduction method on the human $450 \mathrm{~K}$ methylation array to remove tissue specific non-variable CpGs. Clin Epigenetics. 2017;9:11.

46. Solomon O, MacIsaac J, Quach H, Tindula G, Kobor MS, Huen K, et al. Comparison of DNA methylation measured by Illumina $450 \mathrm{~K}$ and EPIC BeadChips in blood of newborns and 14-year-old children. Epigenetics. 2018;13(6):655-664.

47. Joubert BR, Felix JF, Yousefi P, Bakulski KM, Just AC, Breton C, et al. DNA methylation in newborns and maternal smoking in pregnancy: Genome-wide consortium meta-analysis. Am J Hum Genet. 2016;98(4):680-696.

48. Hodyl NA, Roberts CT, Bianco-Miotto T. Cord blood DNA methylation biomarkers for predicting neurodevelopmental outcomes. Genes (Basel). 2016;7(12):117.

49. Spielberger CD. State-Trait Anxiety Inventory for Adults. Manual, Instrument, and Scoring Guide. Palo Alto, CA: Consulting Psychologists Press; 1983.

50. Meades R, Ayers S. Anxiety measures validated in perinatal populations: A systematic review. J Affect Disord. 2011;133(1-2):1-15.

51. Evans K, Spiby H, Morrell CJ. A psychometric systematic review of self-report instruments to identify anxiety in pregnancy. $J$ Adv Nurs. 2015;71(9):1986-2001.

52. O’Connor TG, Ben-Shlomo Y, Heron J, Golding J, Adams D, Glover V. Prenatal anxiety predicts individual differences in cortisol in pre-adolescent children. Biol Psychiatry. 2005;58(3):211-217.

53. Field T, Diego M, Hernandez-Reif M, Figueiredo B, Deeds O, Ascencio A, et al. 
Comorbid depression and anxiety effects on pregnancy and neonatal outcome. Infant Behav Dev. 2010;33:23-29.

54. Rijlaarsdam J, Pappa I, Walton E, Bakermans-Kranenburg MJ, Mileva-Seitz VR, Rippe RCA, et al. An epigenome-wide association meta-analysis of prenatal maternal stress in neonates: A model approach for replication. Epigenetics. 2016;11(2):140-149.

55. Solomon O, Yousefi P, Huen K, Gunier R, Escudero-Fung M, Barcellos LF, et al. Prenatal phthalate exposure and altered patterns of DNA methylation in cord blood. Env Mol Mutagen. 2017;58(6):398-410. 


\section{FIGURES}

Figure 1. Manhattan and quantile-quantile plot showing meta-analytic associations between general anxiety during pregnancy and cord blood DNA methylation (within 15 cohorts, maximum $\mathrm{N}=6686$ mother-child dyads)

Panel A. Manhattan plot (red line indicates the Bonferroni threshold)

Panel B. Quantile-quantile plot $(\lambda=0.962)$

Figure 2. Forest plot showing associations between general anxiety during pregnancy and cord blood DNA methylation for the most significant associations $\left(\mathrm{p}<5 \times 10^{-5}\right)$, across all cohorts with available data, in order of sample size.

\section{TABLES}

Table 1. Measurement of maternal anxiety across the participating studies

Table 2. Characteristics across participating studies

Table 3. General maternal anxiety during pregnancy and cord blood DNA methylation: associations with $\mathrm{p}$-value below $\mathrm{p}<5 \times 10-5$. 